\title{
Effects of intradialytic resistance exercise on systemic inflammation in maintenance hemodialysis patients with sarcopenia: a randomized controlled trial
}

\author{
Zhi-Juan Dong ${ }^{1}$ (D) Hai-Lin Zhang ${ }^{1} \cdot$ Li-Xia Yin $^{2}$
}

Received: 5 November 2018 / Accepted: 10 June 2019 / Published online: 3 July 2019

(c) The Author(s) 2019

\begin{abstract}
Purpose To investigate the effect of intradialytic resistance exercise on inflammation markers and sarcopenia indices in maintenance hemodialysis (MHD) patients with sarcopenia.

Methods Forty-one MHD patients with sarcopenia were divided into an intervention group (group E, $n=21$ ) and a control group (group C, $n=20$ ). Group C patients only received routine hemodialysis care, whereas group E patients received progressive intradialytic resistance exercise with high or moderate intensity for 12 weeks at three times per week (using the weight of the lower limbs and elastic ball movement of the upper limb) on the basis of routine hemodialysis care.

Results After 12 weeks, a significant difference in physical activity status (maximum grip strength, daily pace, and physical activity level), Kt/V, and C-reactive protein was found between groups $\mathrm{E}$ and $\mathrm{C}$. Inflammatory factors (interleukin (IL)-6, IL-10, and tumor necrosis factor(TNF)- $\alpha$ ) increased or decreased more significantly in group E than in group C.

Conclusions This study showed that intradialytic resistance exercise can improve physical activity effectively and reduce microinflammatory reactions even if this simple exercise does not affect the muscle mass in MHD patients with sarcopenia.
\end{abstract}

Keywords Intradialytic resistance exercise $\cdot$ Maintenance hemodialysis $\cdot$ Sarcopenia $\cdot$ Systemic inflammation

\section{Introduction}

Sarcopenia, a newly recognized geriatric syndrome [1], with a prevalence of $4.8-31.0 \%$ has seriously affected the daily lives of people with chronic diseases [2,3]. Disease characteristics are long-term muscle protein imbalance and limited physical activity. Moreover, the amount of protein in the human body accounts for about $20 \%$ of the total muscle. As age advances, the protein intake is relatively insufficient, resulting in protein synthesis, metabolism reduction, and weight loss. In addition, many clinical developments of chronic diseases and malignant tumors occur. These conditions used up protein to varying degrees. Generally,

Hai-Lin Zhang

luckhailin@163.com

1 Department of Nursing, The First People's Hospital of Lianyungang, No. 182, Tongguan North Road, Haizhou District, Lianyungang, Jiangsu, China

2 Department of Hemopurification Center, The First People's Hospital of Lianyungang, Lianyungang, Jiangsu, China functions of the human heart and lungs gradually decrease with age, and muscle mass gradually shrinks, resulting in limited physical activity, further lack of physical activity, and ultimately occurrence of sarcopenia $[4,5]$. The maintenance hemodialysis (MHD) patients as the representative of the sedentary group [6] have a sarcopenia prevalence of $13.7-73.5 \%$ [7, 8]. The situation is grim and needs to be strengthened as sarcopenia is far more prevalent in the ordinary elderly population.

Hemodialysis (HD) provides increased opportunities for endotoxin influx, recurrent infections, and immune activation leading to chronic systemic inflammation [9]. End-stage renal disease (ESRD) patients consequently have a dysfunctional immune system that is both chronically overactivated and anergic [10, 11]. In addition, renal function in patients with ESRD is almost or completely lost, and as the disease worsens, symptoms associated with sarcopenia such as muscle atrophy, decreased muscle strength, and decreased muscle function gradually appear $[12,13]$. The low exercise capacity caused by muscle atrophy is an independent predictor of death in ESRD patients. Both chronic inflammatory state and physical inactivity 
are crucial in the pathogenesis of sarcopenia that is very common in MHD patients. Physical inactivity is a major contributing factor to chronic inflammation and protein energy wasting [14]. Consequently, physical inactivity and chronic inflammatory state can form a vicious cycle in MHD patients with sarcopenia.

Classic interventions to counteract age-related muscle wasting mainly focus on resistance training and/or protein supplementation to overcome anabolic inflexibility from which elderly suffer [15-17]. Although the elderly benefit from these classic interventions, the therapeutic potential of anti-inflammatory strategies is of great interest, as these resistance exercises might add up to the anabolic effect of protein supplementation. Some reports have confirmed the safety and effectiveness of exercise in MHD patients [18-20]. However, no intradialytic exercise is established for MHD patients with sarcopenia. Therefore, in this study, intradialytic resistance exercises were carried out in MHD patients with sarcopenia to investigate whether physical exercise can improve systemic inflammation and sarcopenia indices in such population.

\section{Materials and methods}

\section{Subjects}

The study population was composed of 45 prospectively recruited, voluntary MHD patients with sarcopenia. Prior to enrollment, a total of $341 \mathrm{MHD}$ patients who were treated in the Hemopurification Center, The First People's Hospital of Lianyungang, from May 2017 to July 2017, were selected for the detection of sarcopenia, but only 45 MHD patients with sarcopenia were identified as study subjects. The Institutional Ethics Committee of The First People's Hospital, Lianyungang, Jiangsu, approved the study protocol (2017-2019). Written informed consent was obtained from each participant.

The inclusion criteria were as follows: (1) 18-80 years old, (2) stable dialysis time $\geq 3$ months; (3) no central system disease; (4) can walk independently, no physical disability, muscle strength $\geq$ III; (5) dialysis patients with upper limb internal hemorrhoids; (6) can communicate normally; and (7) voluntarily participate in this study. The exclusion criteria were as follows: (1) pregnant woman; (2) 3 months of bleeding or infection records; (3) cannot perform bioelectrical impedance analysis (BIA) test, such as cardiovascular stent implantation, pacemaker installation, artificial joint replacement or amputation surgery; (4) had other serious complications such as heart failure, serious infection, malignant tumors, etc.; and (5) patients with cognitive impairment and mental illness.

\section{Study design}

Subjects were divided into the intervention group (group $\mathrm{E}, n=23$ ) and control group (group C, $n=22$ ) by the simple randomized method using a random number table (Fig. 1). A study has shown [21] that resistance exercise is the active movement of muscles to overcome external resistance. Compared with aerobic exercise, it focuses on improving cardiopulmonary function. Resistance exercise can improve muscle mass, muscle strength, and muscle function which appropriately target the symptoms of sarcopenia (reduced muscle cross-sectional area, muscle strength, and decreased systolic function [1]). Therefore, only patients in group $\mathrm{C}$ received routine HD care. Patients in group E performed progressive intradialytic resistance exercise with high or moderate intensity for 12 weeks at three times per week (using their own body weight and elastic balls) on the basis of routine HD care.

In the first week, the ankle weight was $0 \mathrm{~kg}$, and quadriceps training board was used to assist the patient in lowintensity resistance training. According to the patient's tolerance, the ankle weight of $+0.5 \mathrm{~kg}$ (single foot) per week until it was $+5 \mathrm{~kg}$ (one foot), with the angle of the training board reduced gradually $\left(150^{\circ}-90^{\circ}\right)$ until it was removed. In the meantime, the untreated hand was holding the elastic ball for $10 \times 10$ performing each step of the upper limb resistance exercise. During the exercise, patients performed a 5-min warm up followed by a 1-2 $\mathrm{h}$ bout of intradialytic resistance exercise: for the one-legraise-and-down exercise, and upper limb bouncing ball movement which exerted pressure on the elastic ball and maximally maintained for $3-5 \mathrm{~s}$ for one cycle and then release it, both complete $10 \times 10$ cycles repeatedly.

During the exercise, a continuous electrocardiography monitor was used to monitor patient's vital signs (blood pressure, heart rate, pulse, oxygen saturation, etc.) and observe the patient's condition. Exercise should be stopped immediately when any of the following occurs [22]: low blood pressure or high blood pressure, over $80 \%$ of the maximum heart rate (maximum heart rate $=220-$ age), Borg score $>15$ points [23], hypoglycemia, dizziness, headache, pallor, chest pain, and exercise-induced breathing difficulties, etc.

\section{Diagnosis of sarcopenia and related symptoms}

The diagnosis of sarcopenia mainly includes declining levels of muscle mass, muscle strength, and physical performance (simultaneously present low levels of muscle mass and muscle strength/muscle function). This study uses the Asia Working Group for Sarcopenia (AWGS) [1] 
Fig. 1 Consort flow chart for study participation

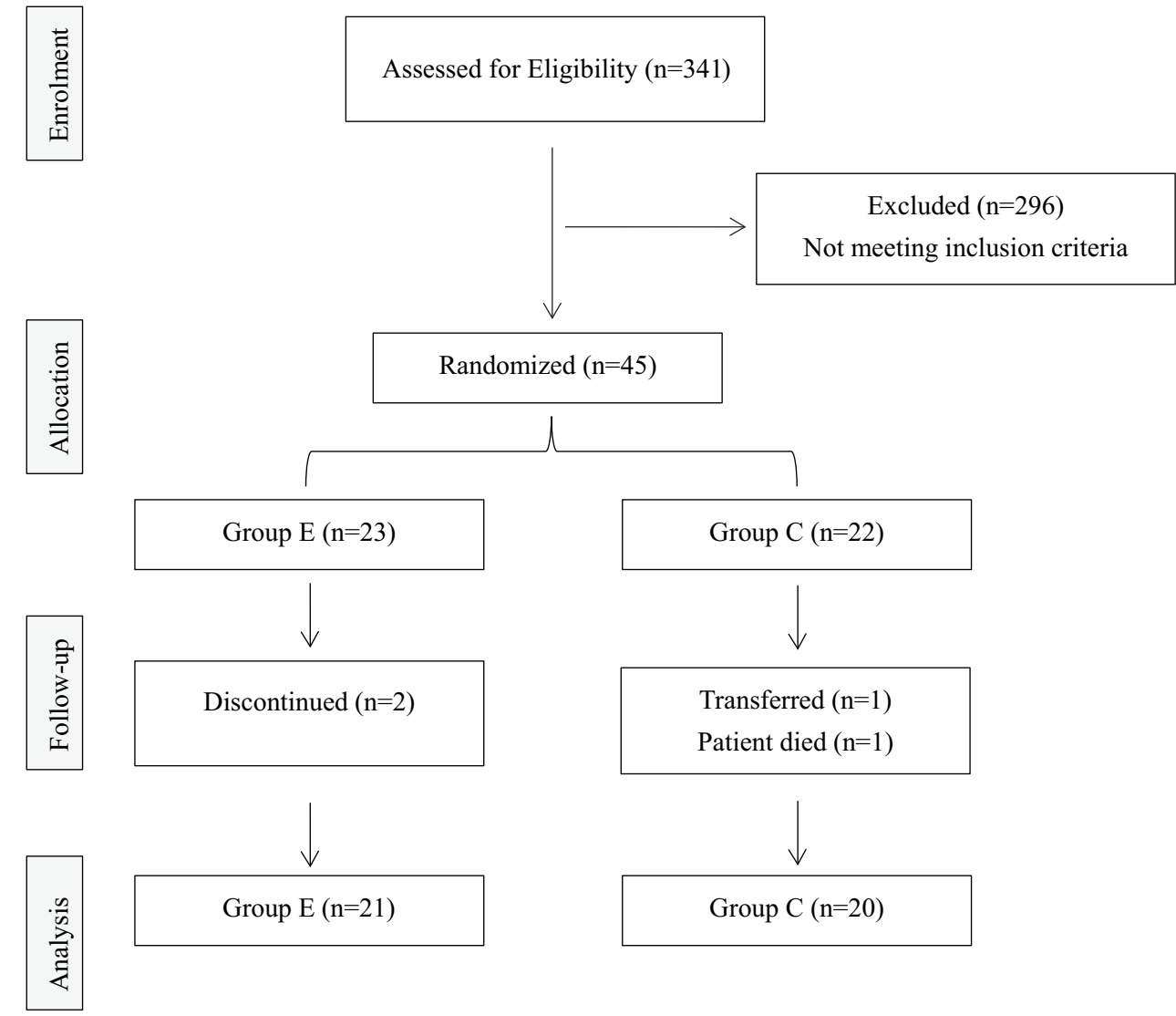

to recommend a diagnostic threshold: BIA skeletal muscle mass index (SMI) (male $<7.0 \mathrm{~kg} / \mathrm{m}^{2}$, female $<5.7 \mathrm{~kg} / \mathrm{m}^{2}$ ), hand grip strength (HGS) (male $<26 \mathrm{~kg}$, female $<18 \mathrm{~kg}$ ), and daily gait speed $(<0.8 \mathrm{~m} / \mathrm{s})$.

On the dialysis day, HGS was measured before the dialysis procedure (the patient stood straight with the limbs naturally sagged [24], three measurements were attempted using an electronic dynamometer (EH101, Xiangshan, China), each time for $5 \mathrm{~s}$, taking the maximum) and daily pace [25] (the patient walked on the ground, the time spent every $6 \mathrm{~m}$ of walking was recorded) using BIA technology [26, 27] (InBody770, InBody, China) to measure the body component-related nutritional indicators (body mass index (BMI), SMI, skeletal muscle mass (SMM), fat-free body weight (FFM), fat-free body mass (FFMI), waist-to-hip ratio (WHR), fat mass (FM), fat mass index (FMI)) within 30-60 min after dialysis on the same day.

\section{Physical activity status}

In this study, we used the international physical activity questionnaire to assess the level of human activity effectively. The questionnaire mainly consists of four parts. According to the evaluation of patient's sitting, walking, cycling, and running ability, weight, etc., patient's physical activity level (light, moderate, and intense) is evaluated: the lower the level, the less the physical activity [28].

\section{Cytokine, albumin, and high-sensitivity C-reactive protein (hs-CRP) measurements}

For the inflammatory modulation analyzes, $8 \mathrm{~mL}$ of venous blood was collected from the antecubital vein into tubes without anticoagulant in pre-HD, immediately after the HD session for both groups (post HD). Serum samples were separated by centrifugation for $5 \mathrm{~min}$ at $1048 \mathrm{~g}(2500 \mathrm{rpm})$, divided into aliquots, and frozen at $-80{ }^{\circ} \mathrm{C}$ for further analysis.

Serum IL-6, IL-10, and TNF- $\alpha$ were measured using enzyme-linked immunosorbent assays. hs-CRP was measured using particle-enhanced immunoturbidimetric assay. Serum albumin was measured using a colorimetric assay.

\section{Statistical analysis}

Statistical analysis was performed using SPSS 23.0 (SPSS Inc., Chicago, IL, USA). Normal measurement data were expressed as mean \pm standard deviation, and $t$ test was used for comparison between groups. Non-normal measurement data were expressed by median and quartile, and nonparametric test was used for comparison between groups. The 
count data usage rate $(\%)$ is expressed as a comparison between groups using the $\chi^{2}$ test or Fisher's exact probability method. Statistical significance of variables was established at the level $P<0.05$.

\section{Results}

In this study, all questionnaires (group $\mathrm{E}, n=21$; group $\mathrm{C}$, $n=20$ ) were returned, and the effectivity rate was $100 \%$. There were no significant differences in the demographic characteristics and disease-related data of the study subjects (Table 1). There were also no differences in the major medical treatments, including antiaggregants, antihypertensive drugs, $\beta$-blockers, statins, and erythropoietin between groups $\mathrm{C}$ and $\mathrm{E}$.

\section{Changes in physical activity and biochemistry data}

There was no significant difference in physical activity and biochemistry data between groups $\mathrm{E}$ and $\mathrm{C}$ before the intervention $(P>0.05)$. After the 12 -week intervention period, the differences were statistically significant in maximal grip strength, daily stride rate, and physical activity level between groups $\mathrm{E}$ and $\mathrm{C}(P<0.05)$. There was no statistically significant difference in neutrophil ratio, hemoglobin, serum creatinine, normalized protein catabolic rate, and subjective global assessment, but there was a statistically significant difference in the patient's Kt/V and nutritional index serum albumin (Table 2).

\section{Changes in muscle-related nutritional indicators}

There were no significant differences in body componentrelated nutritional indicators (BMI, SMI, SMM, FFM, FFMI, WHR, FM, FMI) between groups $\mathrm{E}$ and $\mathrm{C}$ before intervention. Before the intervention, 4 weeks after the intervention, and 12 weeks after the intervention, there were no significant differences in the comparison of the same period between the groups $\mathrm{E}$ and $\mathrm{C}$ (Table 3). BMI and FMI differences were statistically significant only in the comparison between groups (Table 4).

Table 1 Comparison of demographic characteristics and disease-related data of study subjects $(n=41)$

\begin{tabular}{|c|c|c|c|c|c|}
\hline & All subjects $(\%) n=41$ & Group E $(\%) n=21$ & Group C $(\%) n=20$ & $x^{2} / t / Z / F$ & $P$ 值 \\
\hline Age & $60.0(43.0,68.0)$ & $59.0(32.5,66.5)$ & $62.5(50.5,70.0)$ & -1.253 & 0.210 \\
\hline Sex & & & & 1.205 & 0.272 \\
\hline Male & $21(51.2)$ & $9(42.9)$ & $12(60.0)$ & & \\
\hline Female & $20(48.8)$ & $12(57.1)$ & $8(40.0)$ & & \\
\hline Dry weight (kg) & $53.21 \pm 9.36$ & $52.03 \pm 9.26$ & $54.44 \pm 9.55$ & -0.817 & 0.419 \\
\hline Economic status & & & & 0.023 & 0.879 \\
\hline Feel financially difficult & $20(48.8)$ & $10(47.6)$ & $10(50.0)$ & & \\
\hline Feel not financially difficulty & $21(51.2)$ & $11(52.4)$ & $10(50.0)$ & & \\
\hline Marital status & & & & 0.067 & 0.796 \\
\hline Married & $30(73.2)$ & $15(71.4)$ & $15(75.0)$ & & \\
\hline No spouse & $11(26.8)$ & $6(28.6)$ & $5(25.0)$ & & \\
\hline Educational level & & & & 3.027 & 0.463 \\
\hline Elementary school and below & $9(22.0)$ & $3(14.3)$ & $6(30.0)$ & & \\
\hline Junior high school & $3(7.3)$ & $2(9.5)$ & $1(5.0)$ & & \\
\hline High school and secondary School & $23(56.1)$ & $14(66.7)$ & $9(45.0)$ & & \\
\hline College and university & $6(14.6)$ & $2(9.5)$ & $4(20.0)$ & & \\
\hline Dialysis age & $59.0(31.5,86.0)$ & $69.0(31.5,87.5)$ & $57.5(32.5,86.5)$ & -0.157 & 0.876 \\
\hline Primary disease & & & & 2.289 & 0.512 \\
\hline Chronic nephritis & $21(51.2)$ & $9(42.9)$ & $12(60.0)$ & & \\
\hline Diabetic nephritis & $6(14.6)$ & $3(14.3)$ & $3(15.0)$ & & \\
\hline Hypertensive nephritis & $5(12.2)$ & $4(19.0)$ & $1(5.0)$ & & \\
\hline Others & $9(22.0)$ & $5(23.8)$ & $4(20.0)$ & & \\
\hline Complication & & & & 0.196 & 0.658 \\
\hline Yes & $15(36.6)$ & $7(33.3)$ & $8(40.0)$ & & \\
\hline No & $26(63.4)$ & $14(66.7)$ & $12(60.0)$ & & \\
\hline
\end{tabular}


Table 2 Comparison of physical activity status and biochemical data between groups $\mathrm{E}$ and $\mathrm{C}$ before and after intervention $(\bar{x} \pm s)$

\begin{tabular}{|c|c|c|c|c|c|c|}
\hline & \multicolumn{2}{|l|}{ Group E $(n=21)$} & \multicolumn{2}{|l|}{ Group C $(n=20)$} & \multirow[t]{2}{*}{$t / F$} & \multirow[t]{2}{*}{$P$ 值 } \\
\hline & Before & After & Before & After & & \\
\hline Maximum grip strength $(\mathrm{kg})$ & $22.23 \pm 5.27$ & $26.03 \pm 3.85$ & $20.99 \pm 6.05$ & $21.34 \pm 6.16$ & 2.937 & 0.006 \\
\hline Daily pace $(\mathrm{m} / \mathrm{s})$ & $0.97 \pm 0.35$ & $1.16 \pm 0.33$ & $0.98 \pm 0.25$ & $0.94 \pm 0.26$ & 2.305 & 0.027 \\
\hline Physical activity level $(n, \%)$ & & & & & $16.137^{\mathrm{a}}$ & $<0.01$ \\
\hline Mild & $12(57.1)$ & $3(14.3)$ & $15(75.0)$ & $13(65.0)$ & & \\
\hline Moderate & $6(28.6)$ & $6(28.6)$ & $5(25.0)$ & $6(30.0)$ & & \\
\hline Severe & $3(14.3)$ & $12(57.1)$ & $0(0.0)$ & $1(5.0)$ & & \\
\hline Neutrophil ratio (\%) & $65.33 \pm 10.30$ & $66.39 \pm 10.24$ & $69.83 \pm 6.55$ & $71.83 \pm 8.29$ & -1.875 & 0.068 \\
\hline Hemoglobin $(\mathrm{g} / \mathrm{L})$ & $107.10 \pm 21.52$ & $103.43 \pm 18.03$ & $105.95 \pm 19.54$ & $105.00 \pm 20.27$ & -0.262 & 0.795 \\
\hline Serum creatinine $(\mathrm{mg} / \mathrm{dL})$ & $9.17 \pm 2.23$ & $9.62 \pm 0.27$ & $9.36 \pm 2.83$ & $9.60 \pm 3.29$ & 0.028 & 0.978 \\
\hline $\mathrm{Kt} / \mathrm{V}$ & $1.61(1.46,1.95)$ & $1.70 \pm 0.27$ & $1.53(1.45,1.70)$ & $1.47 \pm 0.32$ & 2.464 & 0.018 \\
\hline Serum albumin $(g / L)$ & $37.19 \pm 3.72$ & $39.84 \pm 3.37$ & $37.79 \pm 2.68$ & $36.01 \pm 6.39$ & 2.384 & 0.024 \\
\hline nPCR (g/kg/d) & $1.39 \pm 0.41$ & $1.74 \pm 0.30$ & $1.38 \pm 0.69$ & $1.78 \pm 0.49$ & -0.321 & 0.750 \\
\hline
\end{tabular}

$P$ two groups after the intervention, $K t / V$ dialysis adequacy, $n P C R$ standard protein decomposition rate

${ }^{\text {a }} F$ value

Table 3 Comparison of BIA-related nutritional indicators at different time points before and after intervention in the two groups

\begin{tabular}{|c|c|c|c|c|c|c|}
\hline & \multicolumn{3}{|l|}{ Group $\mathrm{E}(n=21)$} & \multicolumn{3}{|l|}{ Group C $(n=20)$} \\
\hline & Before & 4 weeks & 12 weeks & Before & 4 weeks & 12 weeks \\
\hline BMI & $18.96 \pm 3.08$ & $18.83 \pm 3.07$ & $19.44 \pm 3.26$ & $20.49 \pm 3.41$ & $20.45 \pm 3.25$ & $20.82 \pm 3.41$ \\
\hline SMI & $5.70 \pm 0.80$ & $5.54 \pm 0.70$ & $5.69 \pm 0.84$ & $5.87 \pm 0.69$ & $5.85 \pm 0.69$ & $5.90 \pm 0.69$ \\
\hline SMM (kg) & $21.19 \pm 3.65$ & $20.60 \pm 3.40$ & $21.20 \pm 3.93$ & $21.06 \pm 3.12$ & $21.12 \pm 2.97$ & $21.21 \pm 2.97$ \\
\hline FM (kg) & $10.10(8.05,18.50)$ & $10.80(8.90,18.80)$ & $10.00(8.20,20.55)$ & $14.15(9.13,19.90)$ & $14.35(9.63,19.90)$ & $14.05(11.55,19.75)$ \\
\hline FMI & $3.40(3.15,6.85)$ & $3.80(3.30,7.05)$ & $3.90(3.05,7.45)$ & $5.60(3.28,7.33)$ & $5.60(3.28,7.18)$ & $5.75(4.32,7.92)$ \\
\hline FFM (kg) & $39.66 \pm 6.38$ & $38.59 \pm 5.91$ & $39.77 \pm 7.02$ & $39.37 \pm 5.24$ & $39.05 \pm 4.91$ & $39.68 \pm 4.96$ \\
\hline FFMI & $14.43 \pm 1.71$ & $14.09 \pm 1.54$ & $14.46 \pm 1.88$ & $14.78 \pm 1.25$ & $14.70 \pm 1.31$ & $14.89 \pm 1.24$ \\
\hline WHR & $0.87 \pm 0.06$ & $0.90 \pm 0.07$ & $0.90 \pm 0.07$ & $0.91 \pm 0.07$ & $0.91 \pm 0.07$ & $0.91 \pm 0.08$ \\
\hline
\end{tabular}

$B I A$ bioimpedance analysis, $B M I$ body mass index, $F M$ fat mass, $F M I$ fat mass index, $F F M$ fat-free body weight, $F F M I$ fat-free body mass, $S M I$ skeletal muscle mass index, $S M M$ skeletal muscle mass, $W H R$ waist-to-hip ratio

Table 4 Repeated measures analysis of variance and simultaneous control analysis of BIA-related nutritional indicators in the two groups

\begin{tabular}{|c|c|c|c|c|c|c|c|c|c|c|}
\hline & \multicolumn{6}{|c|}{ Repeated measurement data analysis of variance } & \multicolumn{4}{|c|}{ Contrast analysis results } \\
\hline & $F_{\text {group }}$ & $P$ & $F_{\text {time }}$ & $P$ & $F_{\text {group }} \times$ time & $P$ & $t / Z^{\mathrm{a}}$ & $P$ & $t / Z^{b}$ & $P$ \\
\hline BMI & 5.301 & 0.027 & 0.310 & 0.705 & 0.017 & 0.974 & -1.637 & 0.110 & -1.321 & 0.194 \\
\hline SMI & 1.807 & 0.187 & 0.346 & 0.676 & 0.122 & 0.855 & -1.390 & 0.172 & -0.887 & 0.381 \\
\hline SMM & 0.032 & 0.858 & 0.162 & 0.831 & 0.146 & 0.845 & -0.527 & 0.601 & -0.009 & 0.993 \\
\hline FM & 3.658 & 0.063 & 0.213 & 0.750 & 0.020 & 0.956 & $-1.161^{\mathrm{c}}$ & 0.246 & $-1.461^{\mathrm{c}}$ & 0.144 \\
\hline FMI & 4.369 & 0.043 & 0.202 & 0.763 & 0.031 & 3.000 & $-1.057^{\mathrm{c}}$ & 0.291 & $-1.383^{\mathrm{c}}$ & 0.167 \\
\hline FFM & 0.000 & 0.985 & 0.370 & 0.671 & 0.061 & 0.927 & -0.269 & 0.790 & 0.048 & 0.962 \\
\hline FFMI & 1.990 & 0.166 & 0.488 & 0.593 & 0.106 & 0.877 & -1.367 & 0.179 & -0.874 & 0.387 \\
\hline WHR & 2.017 & 0.163 & 0.123 & 0.851 & 0.136 & 0.839 & -0.569 & 0.573 & -0.798 & 0.430 \\
\hline
\end{tabular}

$B I A$ bioimpedance analysis, $B M I$ body mass index, $F M$ fat mass, $F M I$ fat mass index, $F F M$ fat-free body weight, FFMI fat-free body mass, SMI skeletal muscle mass index, SMM skeletal muscle mass, WHR waist-to-hip ratio

${ }^{\text {a }}$ Two groups after 4 weeks

${ }^{\mathrm{b}}$ Two groups after 12 weeks

${ }^{\mathrm{c}} Z$ value 
Table 5 Comparison of microinflammation status between the two groups

\begin{tabular}{|c|c|c|c|c|c|c|}
\hline & \multicolumn{2}{|l|}{ Group $\mathrm{E}(n=21)$} & \multicolumn{2}{|l|}{ Group C $(n=20)$} & \multirow[t]{2}{*}{$Z$} & \multirow[t]{2}{*}{$P$} \\
\hline & Before & After & Before & After & & \\
\hline $\mathrm{CRP}(\mathrm{pg} / \mathrm{mL})$ & $2.23(1.29,5.24)$ & $2.02(1.40,3.73)$ & $2.91(1.91,8.48)$ & $3.47(1.95,7.10)$ & -2.335 & 0.020 \\
\hline IL-6 (pg/mL) & $7.72(4.95,14.33)$ & $9.43(6.30,12.47)$ & $5.48(3.89,10.06)$ & $7.93(5.61,11.31)$ & -0.757 & 0.449 \\
\hline IL-10 (pg/mL) & $0.82(0.54,0.94)$ & $1.37(1.18,2.00)$ & $0.79(0.52,1.43)$ & $1.21(0.93,1.67)$ & -0.939 & 0.348 \\
\hline TNF- $\alpha(\mathrm{pg} / \mathrm{mL})$ & $1.35(0.59,6.85)$ & $1.26(0.51,8.96)$ & $0.60(0.20,6.59)$ & $4.45(0.35,12.19)$ & -0.665 & 0.506 \\
\hline IL-6/TNF- $\alpha$ & $5.83(1.24,13.30)$ & $8.07(1.91,14.09)$ & $7.73(2.25,17.85)$ & $2.24(0.64,22.73)$ & -1.174 & 0.241 \\
\hline
\end{tabular}

$Z$ and $P$ are compared between the two groups after intervention

$C R P$ C-reactive protein, $I L$ interleukin, $T N F$ - $\alpha$ tumor necrosis factor-alpha

Table 6 Comparison of the mean number of microinflammation factors between the two groups $(\bar{x})$

\begin{tabular}{|c|c|c|c|c|c|c|}
\hline & \multicolumn{2}{|c|}{ Group $\mathrm{E}(n=21)$} & \multicolumn{2}{|c|}{ Group C $(n=20)$} & \multirow{2}{*}{$\begin{array}{l}\text { Difference } \\
\text { between group E }\end{array}$} & \multirow{2}{*}{$\begin{array}{l}\text { Difference } \\
\text { between group } \\
\text { C }\end{array}$} \\
\hline & Before & After & Before & After & & \\
\hline CRP (pg/mL) & 3.71 & 2.79 & 5.86 & 5.24 & -0.92 & -0.62 \\
\hline IL-6 (pg/mL) & 23.23 & 32.49 & 40.24 & 41.23 & 9.26 & 0.99 \\
\hline IL-10 (pg/mL) & 1.22 & 3.4 & 3.83 & 4.55 & 2.18 & 0.72 \\
\hline TNF- $\alpha(\mathrm{pg} / \mathrm{mL})$ & 114.99 & 76.71 & 108.04 & 102.86 & -38.28 & -5.18 \\
\hline IL-6/TNF- $\alpha$ & 10.59 & 30.39 & 10.45 & 10.37 & 19.8 & -0.08 \\
\hline
\end{tabular}

CRP, C-reactive protein; IL, interleukin; TNF- $\alpha$, tumor necrosis factor-alpha

Fig. 2 Comparison of the mean difference in inflammatory factors before and after intervention between groups $\mathrm{E}$ and $\mathrm{C}$

\section{IL-6}

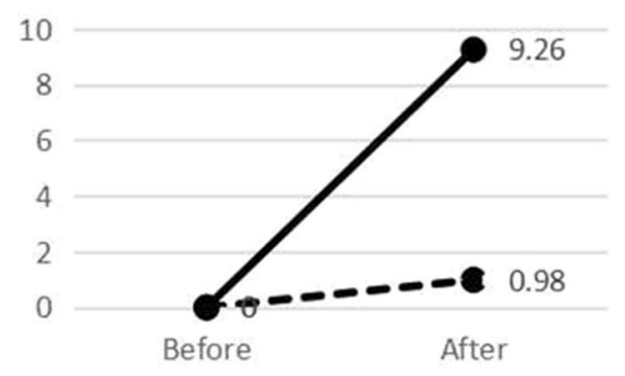

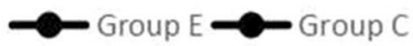

TNF- $\alpha$

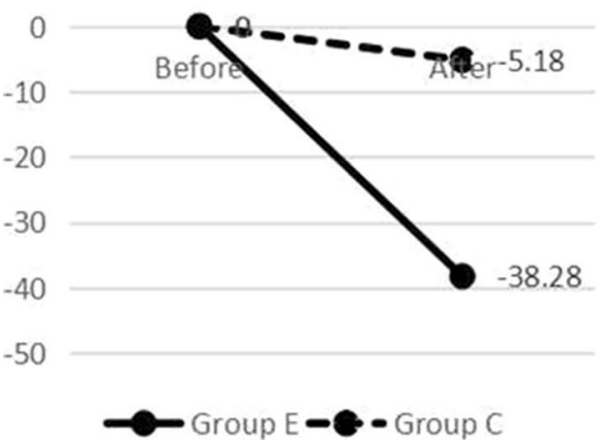

IL-10

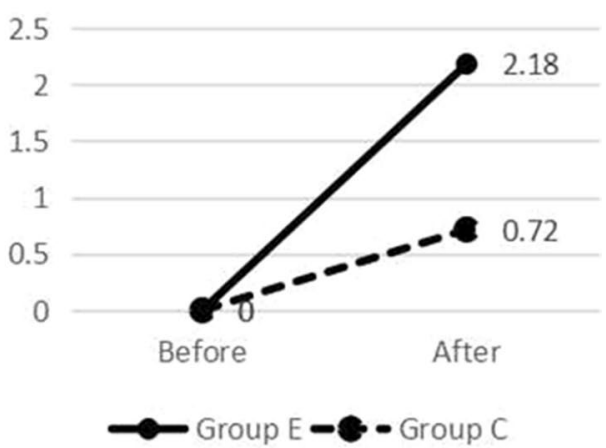

IL-6/TNF- $\alpha$

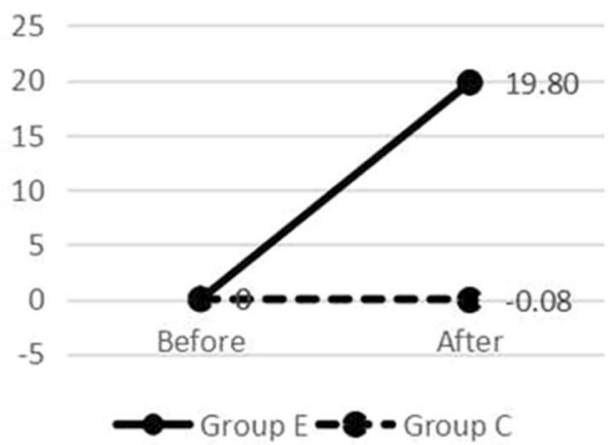




\section{Changes in systemic inflammation}

There were no significant differences in CRP, IL-6, IL-10, TNF- $\alpha$, IL-6/TNF- $\alpha$ between the two groups before intervention $(P>0.05)$. After the intervention, the difference in CRP between the two groups was statistically significant $(P<0.05)$ (Table 5). The mean values were compared between the two groups after the intervention in the course of the data between groups E and C (Table 6). IL-6 and IL-10 as representatives of anti-inflammatory factors were increased after the intervention and the pro-inflammatory factor TNF- $\alpha$ decreased significantly after the intervention (Fig. 2).

\section{Discussion}

The occurrence and development of sarcopenia in MHD patients have been paid attention at home and abroad. In this study, the prevalence of sarcopenia in MHD patients was $13.2 \%$, of which the prevalence rate in 50-year-olds was $43.2 \%$, and the prevalence rate in 60 -year-olds was $55.4 \%$, which is similar to the overall prevalence of sarcopenia in patients with MHD at home and abroad (13.7-73.5\%) [7, 8].

This study focused on the effects of exercise on the dialysis process. Patients not only had high compliance (only two cases experienced falls), but also effectively improve systemic inflammation, dialysis adequacy, and physical activity, thereby improving the quality of life of MHD patients with sarcopenia.

\section{Improvement in systemic inflammation}

MHD patients have been chronically inflamed. In the late 1990s, Zimm et al. [29] indicated that inflammation is closely related to cardiovascular disease, protein energy expenditure, and mortality in chronic kidney disease. In myasthenia, chronic inflammation affects muscle protein breakdown and synthesis through several signaling pathways, resulting in loss of muscle mass, muscle strength, and muscle function [30]. Greiwe et al. [31], based on the fact that muscle contraction can reduce systemic low-level inflammation, hypothesized that exercise can regulate inflammation, trigger hypothalamic-pituitary-adrenal axis and sympathetic nervous system, and stimulate the release of cortisol, adrenaline, and other hormones. A large number of studies have shown that $[17,32,33] \mathrm{CRP}$ is a typical manifestation of acute phase inflammation in human tissues, while IL-6 as an anti-inflammatory cytokine and is resistant to the proinflammatory cytokine TNF- $\alpha$. It is known that excess adipose tissue increases the production of the pro-inflammatory cytokine TNF- $\alpha$ [32]. During exercise, the anti-inflammatory cytokine IL-6, by contracting skeletal muscle fibers, not only increases its production and release, but also stimulates other anti-inflammatory cytokines such as IL-10, and also inhibits the production of the pro-inflammatory cytokine TNF- $\alpha$.

In this study, after the 12-week intervention period, the CRP level in the intervention group was significantly lower than that before the intervention, and the difference between the two groups was statistically significant. It is indicated that the anti-resistance movement during dialysis can reduce CRP. In addition, TNF- $\alpha$ in the intervention group was higher than before intervention and IL- 6 and IL- 10 decresed significantly when compared with that before intervention, consistent with the hypothesis on exercise and inflammation mentioned above. Similar to the results of Moraes et al. [33], there were no statistically significant differences in inflammatory cytokines. The possible causes are as follows: the microinflammation state is a chronic process in which short duration and moderate- to high-intensity events are associated with an overall reduction in circulating levels of inflammatory markers, but do not stimulate IL-6 release [34]; differences in laboratory testing methods used to measure different inflammatory markers; patient's inflammatory cytokines were interfered with dialysis treatment. IL-6 and CRP increased significantly at $1-3 \mathrm{~h}$ after dialysis. After dialysis, IL-6 and CRP levels did not increase. TNF- $\alpha$ levels decreased significantly after dialysis but then returned to the baseline levels [35]; small sample size; and the variability of the muscle mass and endurance of the recruited participants.

\section{Positive impact on patient's dialysis adequacy}

Dialysis adequacy $(\mathrm{Kt} / \mathrm{V})$ is an important indicator of evaluating dialysis effectiveness which used a one-chamber urea kinetic model and reflects the ratio of the volume of urea removed in the dialysis to the total amount of water [36]. Whether MHD patients with dystrophy and dialysis treatment are inseparable, and dialysis treatment leads to waste of muscle protein, abnormal hormone synthesis, metabolic acidosis, etc. [37-40], are physiological and pathological factors for the development of myasthenia. Therefore, it is particularly important to improve the dialysis adequacy. During the dialysis, the movement can make the solute, such as urea in cells, advance into the blood circulation, so that the solute concentration gradient between the chambers is reduced, the imbalance is reduced, and the HD adequacy is further increased [41].

According to the Chinese Clinical Guidelines for Hemodialysis Adequacy (K/DOQI) (2015 Edition) [42], a single dialysis $\mathrm{Kt} / \mathrm{V} \geq 1.2$ is recommended, and $\mathrm{Kt} / \mathrm{V} \geq 1.4$ is better when conditions permit. In this study, the $\mathrm{Kt} / \mathrm{V}$ of both groups was greater than 1.4, indicating that the dialysis effect of this research center is better. After 12 weeks of intervention, the difference of Kt/V between the two groups was statistically significant and indicated that resistance to dialysis can improve dialysis adequacy, consistent with 
Kirkman et al.'s report [43]. On the other hand, the percentage of improvement that occurred in dialysis adequacy was $5.6 \%$ after 12 weeks of intervention, and when compared it with aerobic exercise studies was 1.3\% [14]. We can always prove that exercise can increase blood flow velocity in muscle tissue with high solute content such as uric acid, urea, and creatinine during the dialysis, thereby increasing the transport speed of solute in cells and a large amount of metabolic waste moves into the bloodstream, which helps promote HD. Adequacy can promote urea clearance and phosphate removal in the dialysate effectively.

\section{Improvement of the level of physical activity}

Physical activity refers to various activities that result in a significant increase in energy expenditure due to skeletal muscle contraction [44]; individuals must have sufficient physical activity to meet daily needs. An international multicenter joint research has shown [45] that sedentary lifestyles in MHD patients result in generally lower levels of physical activity. Grip strength and daily pace are simple, widely used indicators of muscle strength assessment, and their baseline values are linearly related to daily physical activity $[24,46]$. Studies by Isoyama et al. [47] reported reduced muscle strength compared with simple muscle mass reduction, which increased the risk of death in MHD patients. Regular resistance exercise is effective in improving muscle strength of these patients. In the study by Olvera et al. [48], MHD patients performed resistance exercise twice a week during dialysis treatment for a total of 12 weeks, and the grip strength increased from 19.6 to $21.2 \mathrm{~kg}$. $(P<0.05)$, similar to the results of Song et al. [49]. In foreign studies, most of the 6-min walking test results replace the daily pace, as the two have the same meaning [25]. Frih et al. [30] divided the MHD patients into the intervention group and control group, in which the intervention group performed the exercise for 4 times a week at 4 months; after the intervention, the grip strength test $(+23.54 \%)$ and 6-min walk test improved $(+15.94 \%)$ in the intervention group. There was a statistically significant difference from the control group. The results in the present study are consistent with the results of the above studies. After the 12-week exercise intervention in the intervention group, the percentage of improvement occurred in maximum grip strength and daily pace was $18.9 \%$ and $19.6 \%$, separately. This shows that the anti-resistance exercise improves the patient's quality of life by directly involving the muscles, improving the muscle strength and self-care ability of the patients.

At the same time, the level of physical activity of the patients increased significantly after the 12 -week intervention, and the proportion of mild physical activity levels decreased from 57.1 to $14.3 \%$. However, there was no significant change in physical activity before and after the intervention in group $\mathrm{C}$, indicating that the progressive intradialytic resistance exercise can improve the physical activity level of the patient and ultimately improve the quality of life. In this study, the reason might be that MHD patients were guided to progressive high-intensity dialysis exercise, making full use of the patient's treatment time, which helped to improve patient's participation during exercise. During the dialysis treatment, the medical staff and rehabilitation staff can monitor changes in patient's condition at any time and adjust the exercise intensity according to the patient's condition to ensure the safety of the exercise and at the same time achieve personalization of the exercise therapy.

\section{Comparison of BIA-related nutritional indicators}

BIA is used commonly for the diagnosis of sarcopenia in clinical practice [50], and its nutritional indicators are highly analyzable. Diagnostic indicators for patients with sarcopenia include muscle mass, muscle strength, and/or physical function. While MHD patients generally suffer from low muscle strength due to prolonged sedentary reasons, the diagnosis of sarcopenia is driven by muscle mass in the MHD group [51]. The improvement of MHD with sarcopenia should be based on changes in muscle mass. A large number of research scholars have used randomized controlled trial to verify whether resistance exercise intervention can improve muscle mass. Kirkman et al. [52] followed 23 MHD patients to perform resistance exercise three times a week for a total of 12 weeks, resulting in a muscle volume of $2822-2906 \mathrm{~cm}^{3}$, which was statistically significant compared with the control group and similar to the results of Olvera et al. [48].

However, in this study, group E performed 12-week highintensity resistance exercise at three times per week, and there was no statistical significance in the muscle qualityrelated indicators (SMI, SMM, FM, FMI, FFM, FFMI, WHR) between groups $\mathrm{E}$ and $\mathrm{C}(P>0.05)$, but there were fluctuations in the values. Consistent with the conclusions of Connie et al. [53], simple resistance exercise does not increase muscle mass. This may be due to the following: participants have a high degree of variability, limited tools and equipment cannot capture patient's physical changes over time, the synthesis and decomposition of muscle protein caused by exercise, short intervention time; and type II error caused by a small sample size of the experimental study.

\section{Conclusions}

Intradialytic resistance exercise with medium to high intensity is a safe, low cost, and efficient approach to improving the muscle status and dialysis effect of MHD patients with 
sarcopenia. Meanwhile, we found simultaneous attenuation of inflammatory responses, which might contribute to these beneficial effects of exercise. Large-scale randomized controlled trials are required to optimize the intradialytic resistance exercise program for MHD patients with sarcopenia.

Acknowledgements The Phase 5 " 521 Project" Scientific Research Project in Lianyungang is acknowledged for grant support.

Funding No funding source or industrial links and affiliations.

\section{Compliance with ethical standards}

Conflict of interest The authors declare that they have no conflict of interest.

Ethical approval We confirm that we have read the journal's position on issues involved in ethical publication and affirm that this report is consistent with those guidelines.

Informed consent Informed consent was obtained from all individual participants included in the study.

Open Access This article is distributed under the terms of the Creative Commons Attribution 4.0 International License (http://creativeco mmons.org/licenses/by/4.0/), which permits unrestricted use, distribution, and reproduction in any medium, provided you give appropriate credit to the original author(s) and the source, provide a link to the Creative Commons license, and indicate if changes were made.

\section{References}

1. Chen L, Liu L, Woo J, Assantachai P, Auyeung T, Bahyah KS, Chou M, Chen L, Hsu P, Krairit O, Lee JSW, Lee W, Lee Y, Liang C, Limpawattana P, Lin C, Peng L, Satake S, Suzuki T, Won CW, Wu C, Wu S, Zhang T, Zeng P, Akishita M, Arai H (2014) Sarcopenia in Asia: consensus report of the Asian Working Group for Sarcopenia. J Am Med Dir Assoc 15(2):95-101

2. Bijlsma Cheng Q, Zheng LL, Zhang ZL (2016) Epidemiology and pathogenesis of sarcopenia. CJOBMR 9(3):228-235

3. Arango-Lopera VE, Arroyo P, Gutierrez-Robledo LM, PerezZepeda MU, Cesari M (2013) Mortality as an adverse outcome of sarcopenia. J Nutr Health Aging 17(3):259-262

4. Cheng Q, Zhu X, Zhang X, Li H, Du Y, Hong W, Xue S, Zhu $\mathrm{H}$ (2014) A cross-sectional study of loss of muscle mass corresponding to sarcopenia in healthy Chinese men and women: reference values, prevalence, and association with bone mass. J Bone Min Metab 32(1):78-88

5. Bijlsma AY, Meskers CG, Ling CH, Narici M, Kurrle SE, Cameron ID, Westendorp RG, Maier AB (2013) Defining sarcopenia: the impact of different diagnostic criteria on the prevalence of sarcopenia in a large middle aged cohort. Age (Dordr) 35(3):871-881

6. Ortiz A, Covic A, Fliser D, Fouque D, Goldsmith D, Kanbay M, Mallamaci F, Massy ZA, Rossignol P, Vanholder R, Wiecek A, Zoccali C, London GM (2014) Epidemiology, contributors to, and clinical trials of mortality risk in chronic kidney failure. Lancet 383(9931):1831-1843
7. Ren H, Gong D, Jia F, Xu B, Liu Z (2016) Sarcopenia in patients undergoing maintenance hemodialysis: incidence rate, risk factors and its effect on survival risk. Ren Fail 38(3):364-371

8. Lamarca F, Carrero JJ, Rodrigues JC, Bigogno FG, Fetter RL, Avesani CM (2014) Prevalence of sarcopenia in elderly maintenance hemodialysis patients: the impact of different diagnostic criteria. J Nutr Health Aging 18(7):710-717

9. Betjes MG (2013) Immune cell dysfunction and inflammation in end-stage renal disease. Nat Rev Nephrol 9(5):255-265

10. Enoki Y, Watanabe H, Arake R, Fujimura R, Ishiodori K, Imafuku T, Nishida K, Sugimoto R, Nagao S, Miyamura S, Ishima Y, Tanaka M, Matsushita K, Komaba H, Fukagawa M, Otagiri M, Maruyama T (2017) Potential therapeutic interventions for chronic kidney disease-associated sarcopenia via indoxyl sulfateinduced mitochondrial dysfunction. J Cachexia Sarcopenia Muscle 8(5):735-747

11. Vaziri ND, Pahl MV, Crum A, Norris K (2012) Effect of uremia on structure and function of immune system. J Ren Nutr 22(1):149-156

12. Fraser S, Bennett PN, Barnard R, Haines T, Ockerby C, Street M, Wang WC, Daly R (2016) Effects of an intradialytic resistance training programme on physical function: a prospective stepped-wedge randomized controlled trial. Nephrol Dial Transplant 31(8):1302-1309

13. Beddhu S, Chen X, Wei G, Raj D, Raphael KL, Boucher R, Chonchol MB, Murtaugh MA, Greene T (2017) Associations of protein-energy wasting syndrome criteria with body composition and mortality in the general and moderate chronic kidney disease populations in the United States. Kidney Int Rep 2(3):390-399

14. Liao M, Liu W, Lin F, Huang C, Chen S, Liu C, Lin S, Lu K, Wu C (2016) Intradialytic aerobic cycling exercise alleviates inflammation and improves endothelial progenitor cell count and bone density in hemodialysis patients. Medicine 95(27):e4134

15. Tomayko EJ, Kistler BM, Fitschen PJ, Wilund KR (2015) Intradialytic protein supplementation reduces inflammation and improves physical function in maintenance hemodialysis patients. J Ren Nutr 25(3):276-283

16. Zdzieblik D, Oesser S, Baumstark MW, Gollhofer A, König D (2015) Collagen peptide supplementation in combination with resistance training improves body composition and increases muscle strength in elderly sarcopenic men: a randomised controlled trial. Br J Nutr 114(8):1237-1245

17. Molsted S, Andersen JL, Eidemak I, Harrison AP, Jørgensen N (2014) Resistance training and testosterone levels in male patients with chronic kidney disease undergoing dialysis. Biomed Res Int 2014:1-7

18. Bessa B, de Oliveira LV, Moraes C, Barboza J, Fouque D, Mafra D (2015) Resistance training in hemodialysis patients: a review. Rehabil Nurs 40(2):111-126

19. Moraes C, Marinho S, Lobo JC, Stockler-Pinto MB, Barros AF, Jacobson LV, Da Nobrega ACL, Rosa MLG, Denise M (2015) Effects of resistance exercise training on acyl-ghrelin and obestatin levels in hemodialysis patients. Ren Fail 37(5):851-857

20. Chao CT, Tang CH, Cheng RW, Wang MY, Hung KY (2017) Protein-energy wasting significantly increases healthcare utilization and costs among patients with chronic kidney disease: a propensity-score matched cohort study. Curr Med Res Opin 33(9):1705-1713

21. Maltais ML, Perreault K, Courchesne-Loyer A, Lagacé J, Barsalani R, Dionne IJ (2016) Effect of resistance training and various sources of protein supplementation on body fat mass and metabolic profile in sarcopenic overweight older adult men: a pilot study. Int J Sport Nutr Exerc Metab 26(1):71-77 
22. Liu YM, Chung YC, Chang JS, Yeh ML (2015) Effects of aerobic exercise during hemodialysis on physical functional performance and depression. Biol Res Nurs 17(2):214-221

23. Compagnat M, Salle JY, Mandigout S, Lacroix J, Vuillerme N, Daviet JC (2018) Rating of perceived exertion with Borg scale in stroke over two common activities of the daily living. Top Stroke Rehabil 25(2):145-149

24. Al SS, Markides KS, Ottenbacher KJ, Raji MA (2004) Hand grip strength and incident ADL disability in elderly Mexican Americans over a seven-year period. Aging Clin Exp Res 16(6):481-486

25. Erlandson MC, Lorbergs AL, Mathur S, Cheung AM (2016) Muscle analysis using pQCT, DXA and MRI. Eur J Radiol 85(8):1505-1511

26. van Vugt JL, Levolger S, de Bruin RW, van Rosmalen J, Metselaar HJ, Ijzermans JN (2016) Systematic review and meta-analysis of the impact of computed tomography-assessed skeletal muscle mass on outcome in patients awaiting or undergoing liver transplantation. Am J Transplant 16(8):2277-2292

27. Guralnik JM, Simonsick EM, Ferrucci L, Glynn RJ, Berkman LF, Blazer DG, Scherr PA, Wallace RB (1994) A short physical performance battery assessing lower extremity function: association with self-reported disability and prediction of mortality and nursing home admission. J Gerontol 49(2):85-94

28. Fan MY, Lü WY, He PP (2014) Calculation method of physical activity level in international physical activity questionnaire. Chin J Epidemiol 35(8):961-964

29. Zimmermann J, Herrlinger S, Pruy A, Metzger T, Wanner C (1999) Inflammation enhances cardiovascular risk and mortality in hemodialysis patients. Kidney Int 55(2):648-658

30. Dalle S, Rossmeislova L, Koppo K (2017) The role of inflammation in age-related sarcopenia. Front Physiol 8:1-17

31. Greiwe JS, Cheng B, Rubin DC, Yarasheski KE, Semenkovich CF (2001) Resistance exercise decreases skeletal muscle tumor necrosis factor alpha in frail elderly humans. FASEB J 15(2):475-482

32. Petersen AMW, Pedersen BK (2005) The anti-inflammatory effect of exercise. J Appl Physiol 98(4):1154-1162

33. Moraes C, Marinho SM, Da Nobrega AC, de Oliveira Bessa B, Jacobson LV, Stockler-Pinto MB, Da Silva WS, Mafra D (2014) Resistance exercise: a strategy to attenuate inflammation and protein-energy wasting in hemodialysis patients? Int Urol Nephrol 46(8):1655-1662

34. Dungey M, Hull KL, Smith AC, Burton JO, Bishop NC (2013) Inflammatory factors and exercise in chronic kidney disease. Int J Endocrinol 2013:569-576

35. Wong J, Davis P, Patidar A, Zhang Y, Vilar E, Finkelman M, Farrington $\mathrm{K}$ (2017) The effect of intra-dialytic exercise on inflammation and blood endotoxin levels. Blood Purif 44(1):51-59

36. Misra M, Nolph KD (2000) Adequacy in dialysis: intermittent versus continuous therapies. Nefrologia 20(3):25-32

37. Dong J, Ikizler TA (2009) New insights into the role of anabolic interventions in dialysis patients with protein energy wasting. Curr Opin Nephrol Hypertens 18(6):469-475

38. Sabatino A, Regolisti G, Karupaiah T, Sahathevan S, Sadu Singh BK, Khor BH, Salhab N, Karavetian M, Cupisti A, Fiaccadori E (2017) Protein-energy wasting and nutritional supplementation in patients with end-stage renal disease on hemodialysis. Clin Nutr 36(3):663-671
39. Maraj M, Kuśnierz-Cabala B, Dumnicka P, Gala-Błądzińska A, Gawlik K, Pawlica-Gosiewska D, Ząbek-Adamska A, MazurLaskowska M, Ceranowicz P, Kuźniewski M (2018) Malnutrition, inflammation, atherosclerosis syndrome (MIA) and diet recommendations among end-stage renal disease patients treated with maintenance hemodialysis. Nutrients 10(1):69

40. Atherton PJ, Smith K (2012) Muscle protein synthesis in response to nutrition and exercise. J Physiol 590(5):1049-1057

41. Rees L (2018) Assessment of dialysis adequacy: beyond urea kinetic measurements. Pediatr Nephrol 26(5):1-9

42. Chinese Medical Association Nephrology Branch Branch Hemodialysis Adequacy Collaboration Group (2015) Guidelines for clinical practice of hemodialysis adequacy in China. Chin J Med 95(34):2748-2753

43. Dl K (2013) Interaction between intradialytic exercise and hemodialysis adequacy. Am J Nephrol 38:475-478

44. Caspersen CJ, Powell KE, Christenson GM (1985) Physical activity, exercise, and physical fitness: definitions and distinctions for health-related research. Public Health Rep 100(2):126-131

45. Avesani CM, Trolonge S, Deleaval P, Baria F, Mafra D, FaxenIrving G, Chauveau P, Teta D, Kamimura MA, Cuppari L, Chan M, Heimburger O, Fouque D (2012) Physical activity and energy expenditure in haemodialysis patients: an international survey. Nephrol Dial Transplant 27(6):2430-2434

46. Buchner DM, Larson EB, Wagner EH, Koepsell TD, de Lateur BJ (1996) Evidence for a non-linear relationship between leg strength and gait speed. Age Ageing 25(5):386-391

47. Isoyama N, Qureshi AR, Avesani CM, Lindholm B, Barany P, Heimburger O, Cederholm T, Stenvinkel P, Carrero JJ (2014) Comparative associations of muscle mass and muscle strength with mortality in dialysis patients. Clin J Am Soc Nephrol 9(10):1720-1728

48. Olvera-Soto MG, Valdez-Ortiz R, López Alvarenga JC, EspinosaCuevas MDLÁ (2016) Effect of resistance exercises on the indicators of muscle reserves and handgrip strength in adult patients on hemodialysis. J Ren Nutr 26(1):53-60

49. Song W, Sohng K (2012) Effects of progressive resistance training on body composition, physical fitness and quality of life of patients on hemodialysis. J Korean Acad Nurs 42(7):947-956

50. van Baar H, Hulshof PJM, Tieland M, de Groot CPGM (2015) Bio-impedance analysis for appendicular skeletal muscle mass assessment in (pre-) frail elderly people. Clin Nutr ESPEN 10(4):147-153

51. Bataille S, Serveaux M, Carreno E, Pedinielli N, Darmon P, Robert A (2017) The diagnosis of sarcopenia is mainly driven by muscle mass in hemodialysis patients. Clin Nutr 36(6):1654-1660

52. Kirkman DL, Mullins P, Junglee NA, Kumwenda M, Jibani MM, Macdonald JH (2014) Anabolic exercise in haemodialysis patients: a randomised controlled pilot study. J Cachexia Sarcopenia Muscle 5(3):199-207

53. Rhee CM, Kalantar-Zadeh K (2014) Resistance exercise: an effective strategy to reverse muscle wasting in hemodialysis patients? J Cachexia Sarcopenia Muscle 5(3):177-180

Publisher's Note Springer Nature remains neutral with regard to jurisdictional claims in published maps and institutional affiliations. 